\title{
A vacuum-driven rubber-band gripper
}

\author{
Ko Yamada ${ }^{1}$ and Takashi Mitsuda ${ }^{2 *}$ (B)
}

\begin{abstract}
Robotic grippers that gently handle objects of various shapes are required for various applications these days. Conventional finger-shaped grippers are multifunctional and can grip various objects; however, grasping an item without slippage requires planning the positioning of the fingers at appropriate locations on the item. Hence, a ring-shaped soft gripper that coils itself around objects like a rubber band is suggested in this paper. The proposed gripper comprises a soft tube containing laminated sponges interleaved with plastic sheets. Evacuation of the air within the sponges shrinks them and decreases the diameter of the ring, thereby allowing the gripper to firmly hold objects. The gripper is therefore flexible enough to coil around objects of various shapes without gaps. Furthermore, the rigidity of the compressed sponges inside the gripper prevents wobbling of the gripped objects. The air within the gripper can be used to adjust the gripping force. The minimum diameter of the gripper after evacuating the air within the sponges is approximately one-fourth of the original diameter. Thus, the proposed gripper is expected to be used in various applications as it automatically conforms to the different shapes while simply gripping objects gently and securely.
\end{abstract}

Keywords: Pneumatic actuator, Flexible structure, Soft robot, Gripper, Robot hand, Vacuum-driven gripper

\section{Introduction}

Robotic grippers that can handle objects automatically without manual intervention or human assistance are essential for mechanization. In recent years, the demand for grippers that can gently handle items of various shapes has increased. However, it is challenging for conventional grippers comprising hard parts and motors to hold fragile objects, such as food items, without breaking or slippage. In this context, there is a growing interest in soft robots [1,2], which are composed of flexible materials. Many grippers have been designed and developed with flexible fingers that can be bent by manipulating the internal air pressure [3-6]. A few of these have even been commercialized $[7,8]$. Recently, flexible pneumatic grippers capable of adjusting their own stiffness have been developed [9-13]. However, finger-shaped grippers require fine control of the finger movements

\footnotetext{
${ }^{*}$ Correspondence: mitsuda@is.ritsumei.ac.jp

${ }^{2}$ College of Information Science and Engineering, Ritsumeikan University, Kusatsu-shi, Japan

Full list of author information is available at the end of the article
}

or appropriate positioning of the fingers to prevent the object from slipping through the them.

In contrast, grippers without fingers have also been proposed. A universal gripper [14] grasps an object by holding it using a flexible bag containing particles and stiffening the bag by manipulating its internal vacuum pressure to cause jamming transition of the particles [15]. In the origami magic ball [16], an object is placed within a conical gripper and grasped by shrinking the cone via changes to the internal vacuum pressure; such grippers do not require precise control for grasping objects. However, the former must be pressed against the object to be held, which is not suitable for fragile items, while the latter can only grasp objects with circular cross-sectional shapes. We therefore develop a gripper that can grasp objects of various shapes without pressing them; the proposed ring-shaped gripper encircles the object like a rubber band by evacuating the air within. This gripper can deform along the surface of an object without any gaps even when the object's cross section is non-circular. Gaps between an object and the gripper's surface decreases the contact area; this affects the gripper's stability when holding objects. In addition, a smaller contact area increases 
the contact pressure; such pressure can deform flexible objects. Therefore, when objects (such as, food items or fragile objects) are grasped by the gripper, they should be grasped gently by applying low contact pressure.

The vacuum-driven ring-shaped gripper developed in this study is novel. Although, ring-shaped grippers have been developed by Bridgestone Corporation [17] and Wang et al. [18], their grippers are operated using compressed air. It comprises a hard cylinder and rubber bag. The flexible bag when inflated by compressed air wraps around an item which is inside the cylinder. The cylinder does not change its shape; therefore this could pose an obstacle for observing the state of gripping of the item inside. In contrast, the gripper developed in this study changes its shape according to the item being gripped. Therefore, the grasping state is observable, and the gripper is adequate for operations in narrow spaces.

In this paper, we first present the design of the gripper. Then, the experimental results of a telescopic actuator having the same internal structure as the gripper, which was developed to optimize the gripper design, are presented. Next, we describe the mechanical properties of the developed gripper, including its deformation characteristics, ability to encircle various objects, and gripping force. Finally, we present its attachment method to a robotic hand and performance for handling various objects.

\section{Design}

\section{Overview}

A sponge sealed within a flexible airtight bag shrinks when the air in the bag is evacuated. We first developed a telescopic actuator, as shown in Fig. 1, using this property. Rectangular sponges (INOAC Corp., urethane foam, density $25 \mathrm{~kg} / \mathrm{m}^{3}$ ) and polypropylene sheets (thickness $0.15 \mathrm{~mm}$ ) were alternately laminated and placed within a bag. When the air inside the bag is evacuated, the sponges contract and create dents between the polypropylene sheets, as shown in Fig. 1. This contraction of the sponges shorten the actuator along the direction of its long axis. The length of the actuator varies with the internal vacuum pressure because the compressive force of the pressure and restoring force of the sponges determine the amount of shortening. Based on this mechanism, we developed a ring-shaped gripper that shrinks when the inside air is evacuated, as shown in Fig. 2. As described in the following sections, the gripper is flexible and can encircle objects of various shapes. The internal vacuum pressure in the gripper can be used to adjust

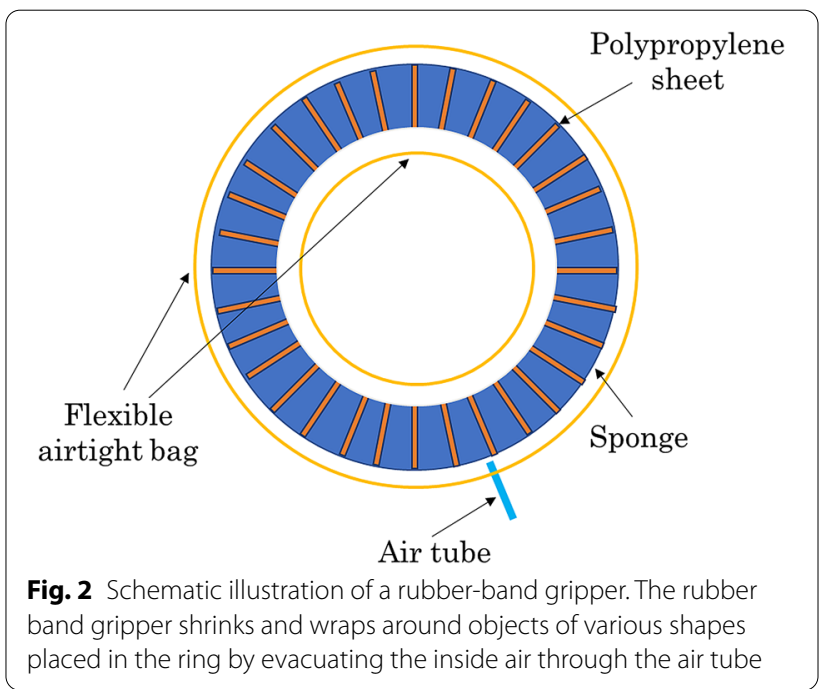

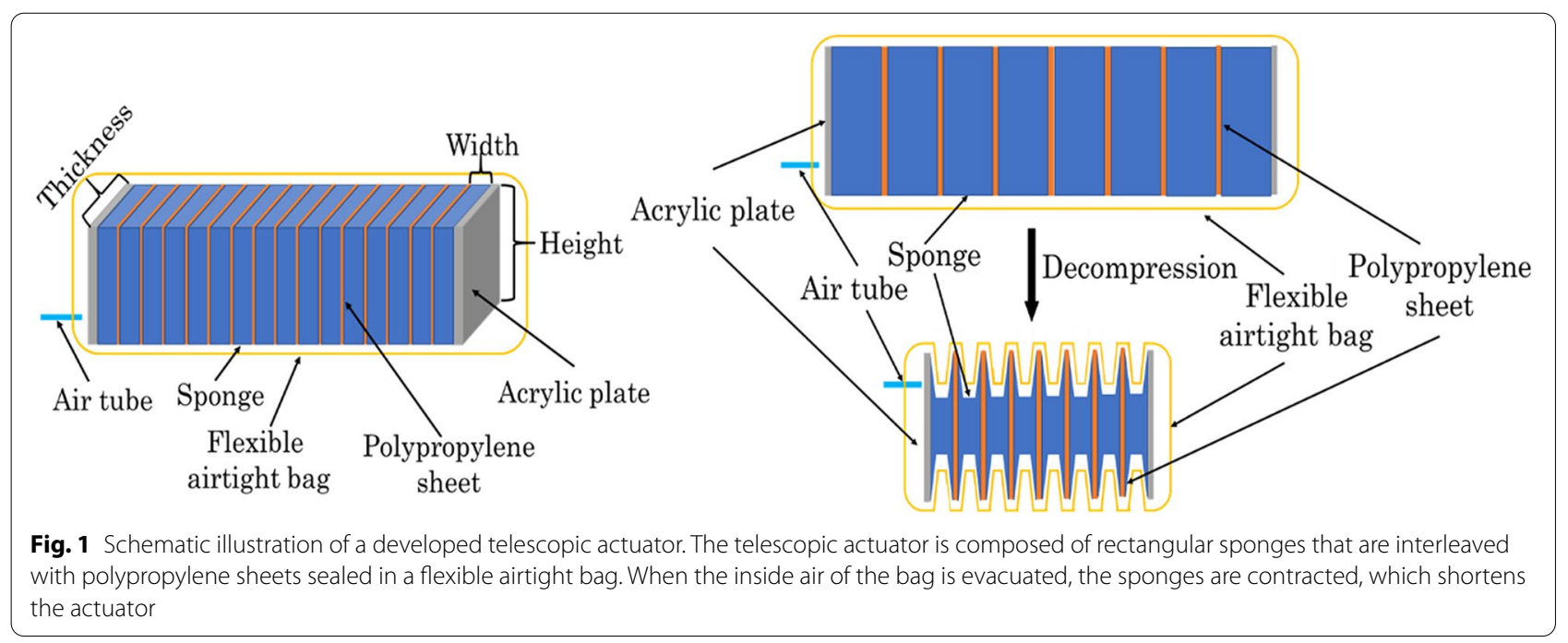


the tightening force. When the internal air pressure is returned to atmospheric pressure, the resilience of the sponges restores the gripper to its original shape and releases the gripped object.

As alternatives to the developed actuator, bellows can be used as pneumatically driven telescopic actuators. However, such bellows must be embedded with hard rings to prevent collapsing when the interior air is evacuated. This is not desirable for gripping fragile items because the hard rings may come into contact with the item, which could distort the flexibility of the gripper's cross-sectional shape. In the developed gripper, the stiffness of the contracted sponge moderately prevents the gripper from being crushed under atmospheric pressure, and the stiffness of the interleaved polypropylene sheets is small. Therefore, the gripper is flexible and can fit to the surfaces of various objects. Contrarily, a gripper made of bellows cannot stably grasp objects because its flexibility is retained even after grasping objects. Increasing the internal vacuum pressure enables the gripper to be rigid; however, the contact pressure on the gripped object may also increase. Further, the developed gripper has increased rigidity after grasping the objects owing to the compressed sponges. Therefore, the proposed gripper can grasp objects stably even though it is composed of soft materials and very flexible in the resting state.

\section{Relationship between shrinkage ratio of telescopic actuator and sponge shape}

The amount of shrinkage of the gripper after evacuation of air varies depending on the shape of the sponges. To increase the range of object sizes that can be grasped by the gripper, the maximum shrinkage ratio, defined as the ratio of decrease in the inner diameter of the ring to its initial inner diameter, should be large. To optimize the internal structure of the rubber-band-like gripper, we investigated the relationship between the shape of the rectangular parallelepiped-like sponges and shrinkage ratio of the telescopic actuator.

The width, height, and thickness of the sponge are defined as shown in Fig. 1. We investigated the effects of the heights and widths of the sponges on the shrinkage ratio along the long axis of the actuator by fixing their thickness to $30 \mathrm{~mm}$. Sponge samples of 15 sizes with different heights $(30,40$, and $50 \mathrm{~mm})$ and widths $(10,20,30$, 40 , and $50 \mathrm{~mm}$ ) were prepared; then, three sponges of each type were connected in series with interleaved polypropylene sheets and encased in a natural rubber sheath of thickness $0.25 \mathrm{~mm}$. The distance between the polypropylene sheets (i.e., width of the sponge) was measured when the internal air pressure was decreased to $-75 \mathrm{kPa}$.

Figure 3 shows the relationship between the width of the sponge in the initial state and the shrinkage ratio at

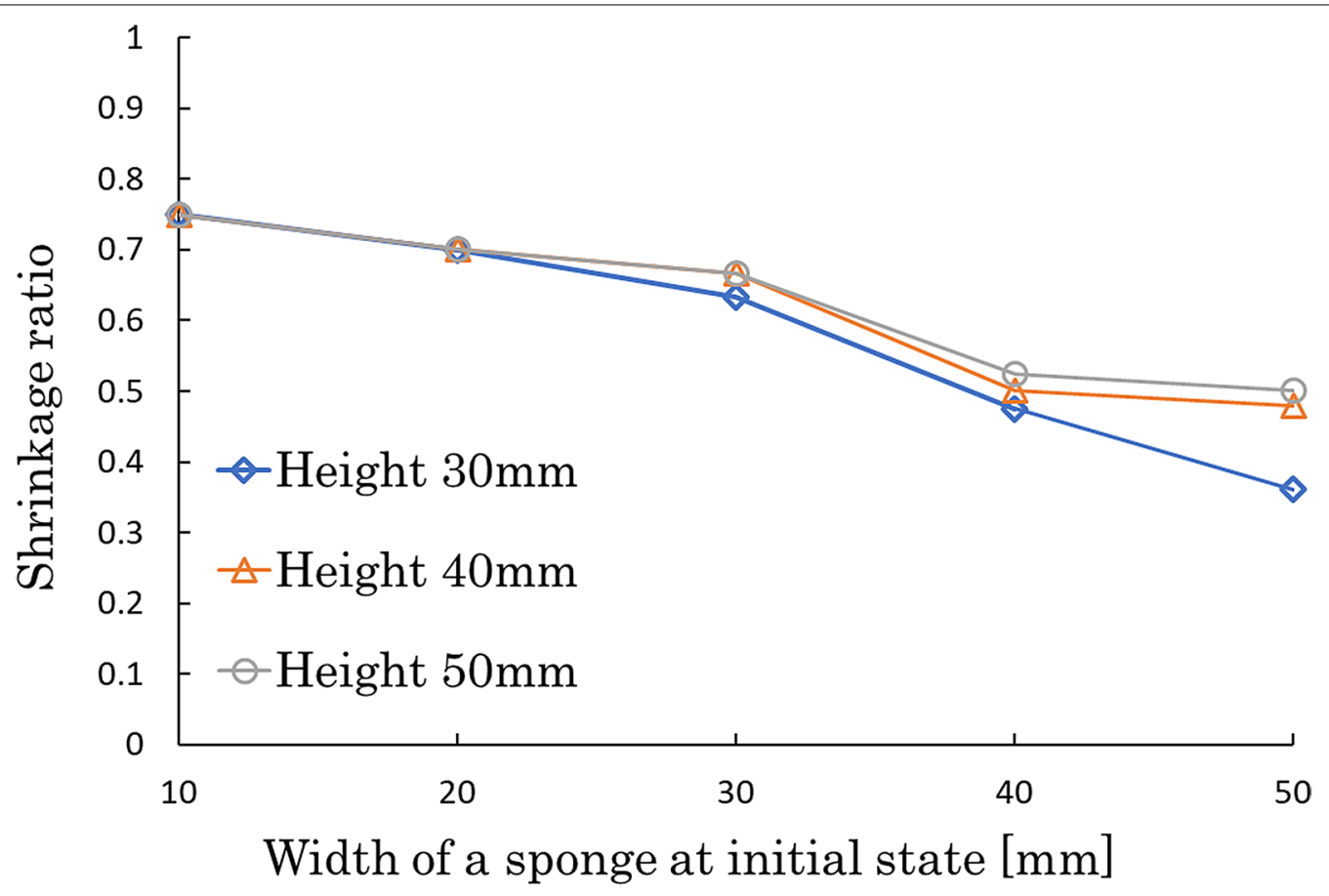

Fig. 3 Relationship between the width of a sponge at the initial state and shrinkage ratio when the inside air pressure of the actuator is - $75 \mathrm{kPa}$. The shrinkage ratio was smaller for thinner sponges. The height of the sponges did not affect the shrinkage ratio when the width of the sponge was small 
$-75 \mathrm{kPa}$ for each height; the smaller the width of the sponge, the greater is the shrinkage ratio. When the width of the sponge is small, the height has little effect on the shrinkage ratio. Thus, a greater shrinkage ratio of the actuator may be obtained when the width of the sponge is less than $10 \mathrm{~mm}$. However, it is difficult to build a gripper with very thin sponges laminated together; hence, in the present study, sponges of width $10 \mathrm{~mm}$ are used to build the gripper.

\section{Relationship between length of the telescopic actuator and internal air pressure}

We examined the length of the telescopic actuator and its relation to the internal air pressure. Ten sponges of height $30 \mathrm{~mm}$, thickness $30 \mathrm{~mm}$, and width $10 \mathrm{~mm}$ were connected in series with interleaved polypropylene sheets and encased in a natural rubber sheath of thickness $0.25 \mathrm{~mm}$. Wooden pieces of height $30 \mathrm{~mm}$, thickness $30 \mathrm{~mm}$, and width $12 \mathrm{~mm}$ were attached to both ends of the connected sponges instead of acrylic plates to fix the actuator to a measuring instrument. Figure 4 shows the relationship between the length of the actuator, excluding the outer membrane and wooden pieces (i.e., length of the connected sponges), and the internal air pressure. The actuator was compressed significantly from atmospheric pressure to approximately $-10 \mathrm{kPa}$ and then further contracted gradually. When the air inside the actuator was evacuated, the ends of the sponges were bent, causing dents between the polypropylene sheets, as shown in Fig. 1. This deformation and compression properties of the sponges thus affect the shortening mechanism of the actuator. This rapid shortening of the actuator at low vacuum pressure therefore enables the gripper to grasp objects gently, as demonstrated in the following sections.

\section{Contraction force of the telescopic actuator}

The rubber-band-like gripper encircles an object by contraction forces generated by the decreasing internal vacuum pressures. After wrapping around the object, the contraction forces generate a wrapping force on the object (i.e., the force perpendicular to the contact surface). To investigate this wrapping force of the gripper, we examined the relationship between the contraction force of the telescopic actuator and its length. The contraction force was measured with a universal testing machine (UTM; IMADA Co., Ltd., MX2-500 N) and a force gauge (IMADA Co., ZTA-200 N) according to the following procedure.

First, one end of the actuator was fixed to the base of the UTM, and the internal air pressure of the actuator was adjusted to the target value, thereby contracting the actuator. Next, the other end of the contracted actuator was fixed to the force gauge after adjusting the position of the force gauge to avoid pulling the actuator. Then, the contraction force was measured after reducing the internal air pressure to $-75 \mathrm{kPa}$. Further, the

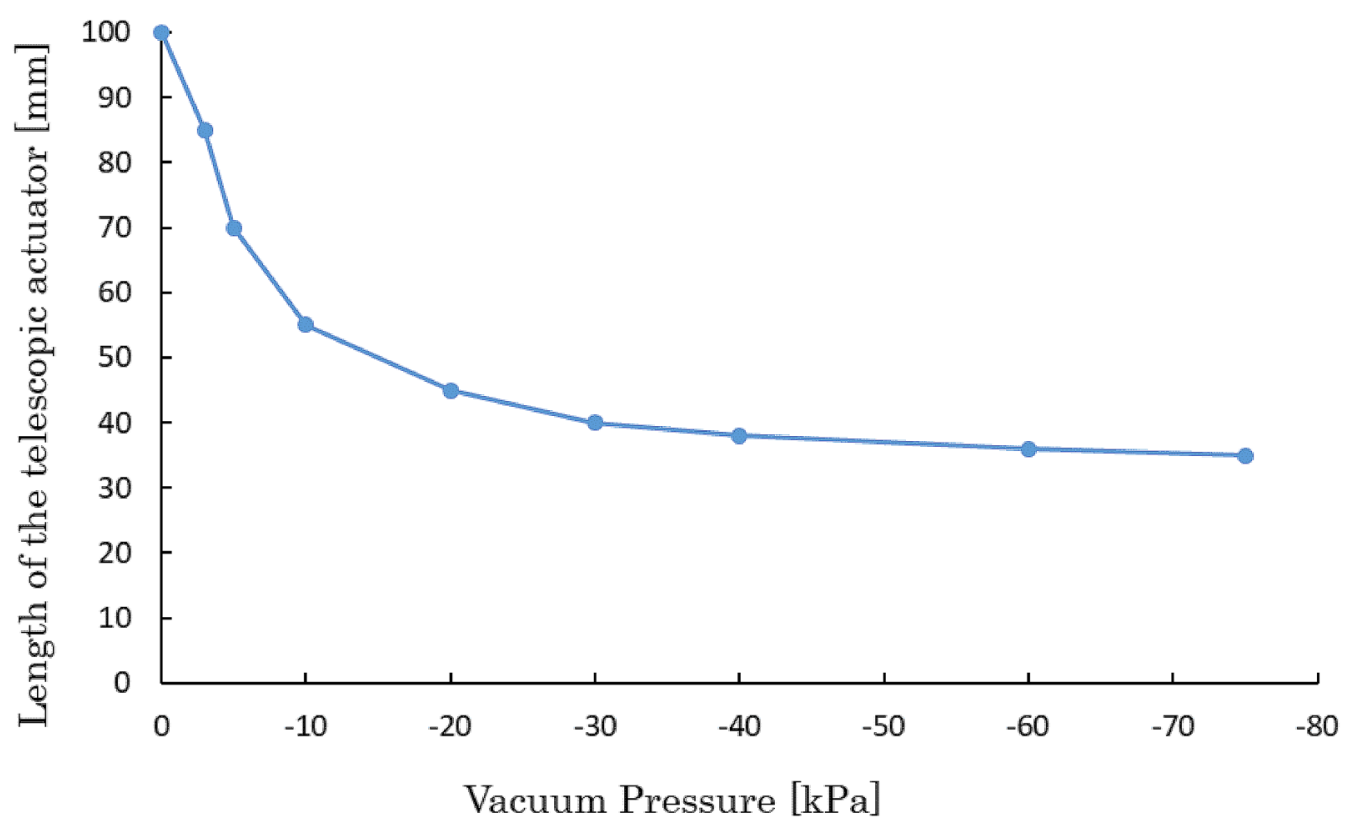

Fig. 4 Relationship between the length of the telescopic actuator and internal air pressure. The telescopic actuator contracted significantly from atmospheric pressure to approximately $-10 \mathrm{kPa}$ and then contracted gradually. The length of the telescopic actuator does not include that of the membrane and the wooden pieces attached to both ends of the actuator 
contraction forces were measured for various lengths of the actuator by adjusting the internal air pressures to $0,-3,-5,-10,-20,-30,-40$, and $-60 \mathrm{kPa}$. Three measurements were obtained for each length of the actuator, and the averages were calculated. Because the contraction force is a result of the difference between the internal air pressure and the pressure when affixed to the force gauge, it is assumed that the contraction force is greater when the length of the actuator is closer to its natural length than when compressed.

Figure 5 shows the relationship between the length of the telescopic actuator and contraction force. The contraction force was smaller for a shorter length of the actuator, which is in agreement with the inference. The nearly constant contraction force from 45 to $85 \mathrm{~mm}$ length was attributed to the fact that the length of the telescopic actuator changed significantly from atmospheric pressure to approximately $-10 \mathrm{kPa}$. When the length of the actuator was in this range, the change in the internal air pressure was small. Therefore, the contraction force, which is be proportional to the difference between the initial air pressure and vacuum pressure during measurement (i.e., $-75 \mathrm{kPa}$ in this experiment), did not change significantly. The increase in the contraction force with length change from 85 to $100 \mathrm{~mm}$ can be attributed to the change in the crosssectional area owing to the indenting of the sponges, as mentioned earlier.
Figure 6 shows the relationship between the contraction force and difference between the internal air pressure when the actuator is fixed to the instrument and pressure during measurement $(-75 \mathrm{kPa})$; the contraction force is observed to be almost proportional to the pressure difference. The steep increase in the contraction force for pressure differences exceeding $70 \mathrm{kPa}$ (i.e., when the length of the actuator was close to its natural length) was owed to the change in the cross-sectional area of the actuator.

The contraction force of the telescopic actuator thus varies with length, unlike pneumatic cylinders. However, the contraction force is almost constant except when the length of the actuator is close to its longest and shortest values. Therefore, when the actuator is used as a ring gripper, the wrapping force is approximately constant regardless of the object size, except when the size is close to the maximum or minimum inner diameter of the ring.

\section{Material of the outer membrane for the rubber-band gripper}

To increase the shrinkage ratio of the rubber-band gripper, it is preferable to have a flexible outer membrane that does not interfere with the contraction of the sponge. According to beam theory, the bending stiffness is determined by the product of Young's modulus and second moment of area. Therefore, to select a suitable outer membrane for the gripper ring, three grippers

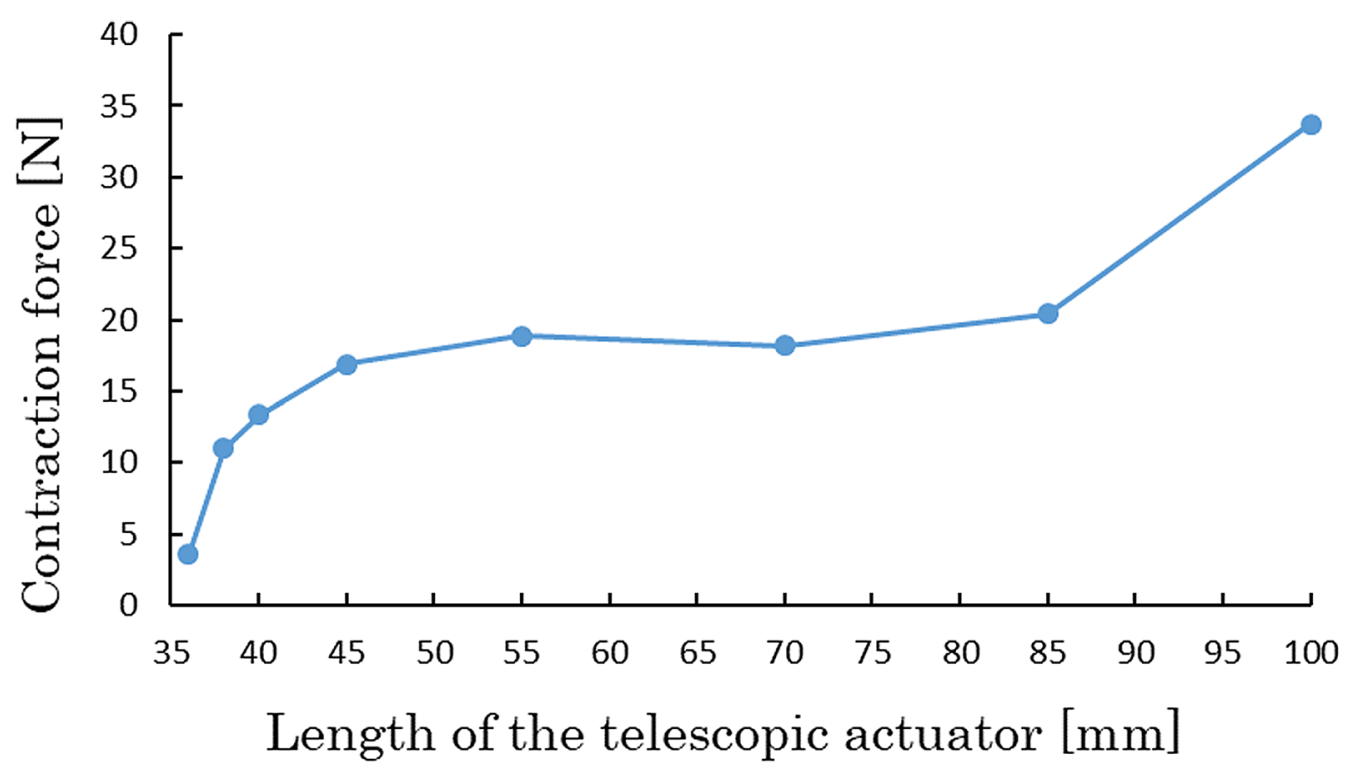

Fig. 5 Relationship between the contraction force of the telescopic actuator when the inside air pressure is $-75 \mathrm{kPa}$ to the length of the actuator. The contraction force was almost constant when the length was in the range of $45-85 \mathrm{~mm}$, which was due to the fact that the telescopic actuator changed its length significantly from atmospheric pressure to approximately $-10 \mathrm{kPa}$. When the length of the actuator was in this range, the inside air pressure was nearly constant, resulting in a constant contraction force. A steeper increase over $85 \mathrm{~mm}$ could be attributed to the change in the cross-sectional area by dents on the surface of the actuator 


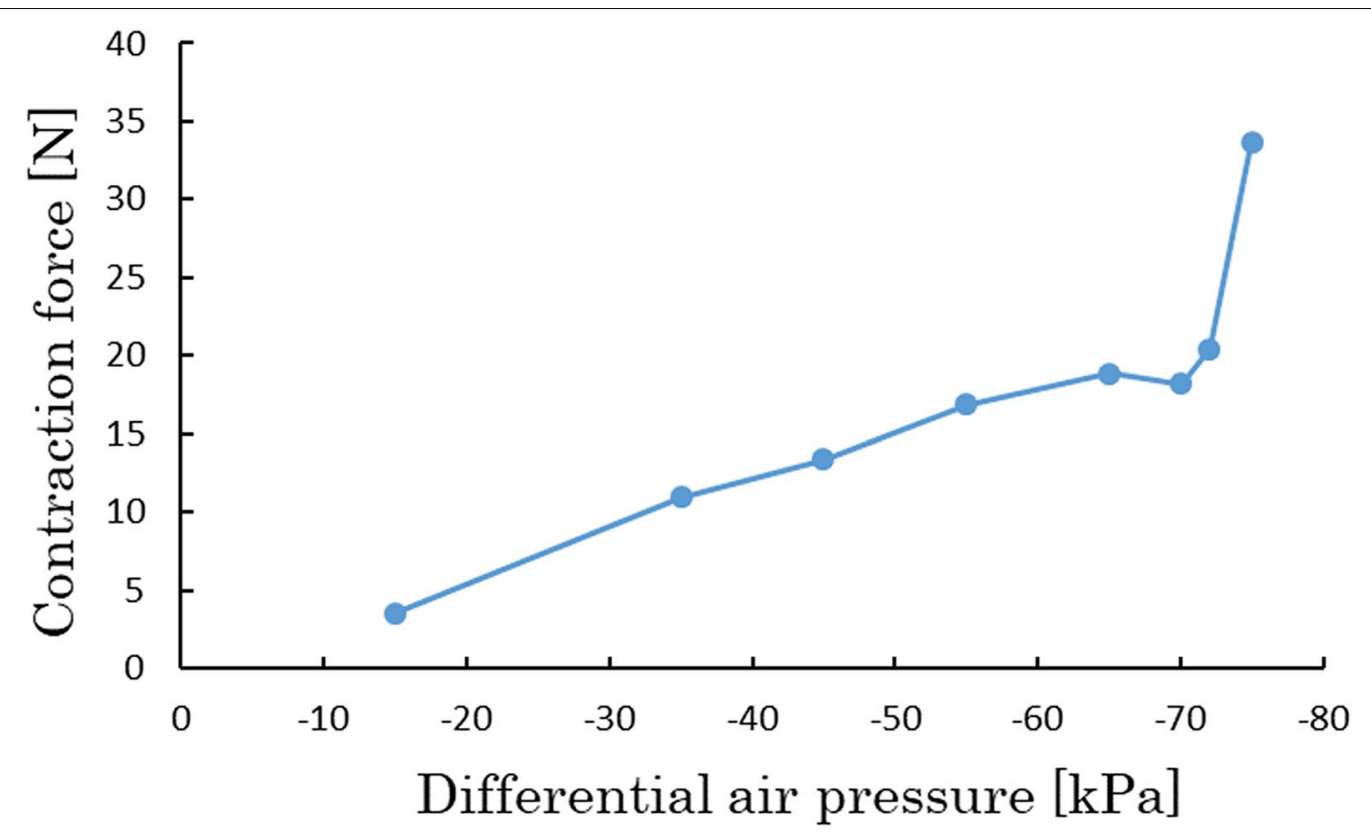

Fig. 6 Relationship between the contraction force and difference between the inside air pressure when the actuator is fixed to the instrument and that at measurement $(-75 \mathrm{kPa}$ ). The contraction force was almost proportional to the differential air pressure. The steep increase over $-70 \mathrm{kPa}$ could be attributed to the change in the cross-sectional area by dents on the surface of the actuator

Table 1 Thickness, Young's modulus, and bending stiffness of each material

\begin{tabular}{llll}
\hline Material & Thickness [mm] & $\begin{array}{l}\text { Young's } \\
\text { modulus } \\
{[\mathrm{MPa}]}\end{array}$ & $\begin{array}{l}\text { Bending } \\
\text { stiffness } \\
{\left[\mathrm{Nm}^{2}\right]^{\mathbf{a}}}\end{array}$ \\
\hline Cotton-lined PVC film & 0.3 & 185 & $1.3 \times 10^{-5}$ \\
Polyethylene tarpaulin & 0.07 & 402 & $3.5 \times 10^{-7}$ \\
Natural rubber & 0.25 & 3 & $1.2 \times 10^{-7}$ \\
\hline
\end{tabular}

a Theoretical value for a rectangular cross-sectional beam of width $30 \mathrm{~mm}$

with different outer membranes of varying thicknesses and Young's moduli were developed. The grippers each enclosed 60 sponges of height $30 \mathrm{~mm}$, thickness $30 \mathrm{~mm}$, and width $10 \mathrm{~mm}$, which were glued together in a circle with interleaved polypropylene sheets of thickness $0.15 \mathrm{~mm}$. The initial inner diameter of each gripper was $150 \mathrm{~mm}$.

Table 1 lists the materials of the outer membrane used in the grippers. The bending stiffness values in the table were estimated according to beam theory. A cotton-lined poly(vinyl) chloride (PVC) film was chosen as the outer membrane because the PVC film without lining deformed plastically even under small forces. The cotton lining therefore increases the Young's modulus and prevents plastic deformation. The polyethylene tarpaulin used in the experiment was a plain weave of polyethylene tape of width $2-4 \mathrm{~mm}$, and the bending
Table 2 Relationship between outer membrane material and shrinkage ratio

\begin{tabular}{ll}
\hline Material & $\begin{array}{l}\text { Shrinkage } \\
\text { ratio }^{\mathbf{a}}\end{array}$ \\
\hline Cotton-lined PVC film & 0.7 \\
Polyethylene tarpaulin & 0.8 \\
Natural rubber & 0.8 \\
\hline
\end{tabular}

${ }^{\text {a }}$ Shrinkage ratio is the quotient of decrease in inner diameter divided by the inner diameter before shrinkage

stiffness of the tarpaulin shown in Table 1 is equivalent to that of a polyethylene film of the same thickness. Therefore, the bending stiffness of the tarpaulin may be smaller than this estimate.

Table 2 shows the shrinkage ratio when the interior of each gripper was depressurized to $-75 \mathrm{kPa}$. The cotton-lined PVC film having the highest bending stiffness showed the least shrinkage ratio. The shrinkage ratios of the polyethylene tarpaulin and natural rubber were almost equal. However, the gripper using the polyethylene tarpaulin required a longer time to return to its original state when released from airtightness. In contrast, the gripper using the natural rubber membrane returned to its original state quickly. The resilience of the natural rubber membrane might thus shorten the expansion time of the gripper. Accordingly, we adopted 
natural rubber for the outer membrane of the proposed gripper.

\section{Experiments}

In this section, the wrapping performance of the gripper with natural rubber membrane is described along with a method of mounting the device to a robot arm.

\section{Relationship between internal air pressure and inner diameter of the gripper}

The relationship between the inner diameter of the gripper and its internal air pressure was investigated. The inner diameter of the gripper was measured as the internal pressure was varied from 0 to $-10 \mathrm{kPa}$ in increments of $1 \mathrm{kPa}$, from -10 to $-70 \mathrm{kPa}$ in increments of $10 \mathrm{kPa}$, and at $-75 \mathrm{kPa}$. Figure 7 shows the deformations of the gripper, and Fig. 8 shows the relationship between the internal air pressure and shrinkage ratio. The shrinkage ratio of the gripper rapidly increased with increase in the vacuum pressure up to approximately $-10 \mathrm{kPa}$, as with the telescopic actuator, and then gradually increased. At vacuum pressures beyond $-30 \mathrm{kPa}$, the inner diameter changed very slightly.

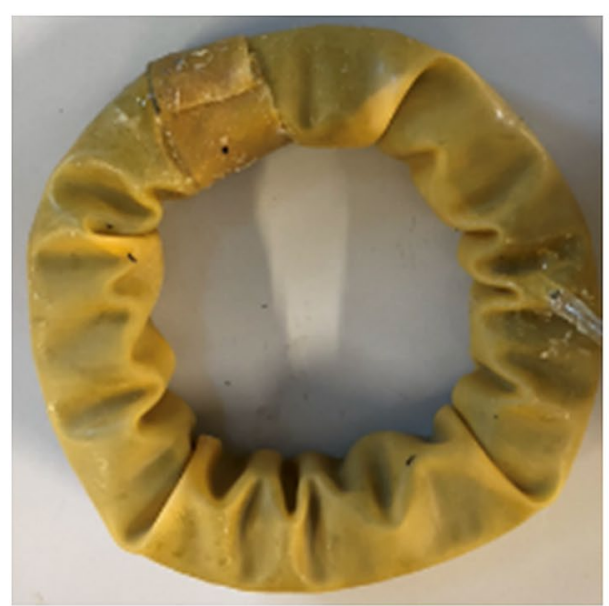

$0 \mathrm{kPa}$

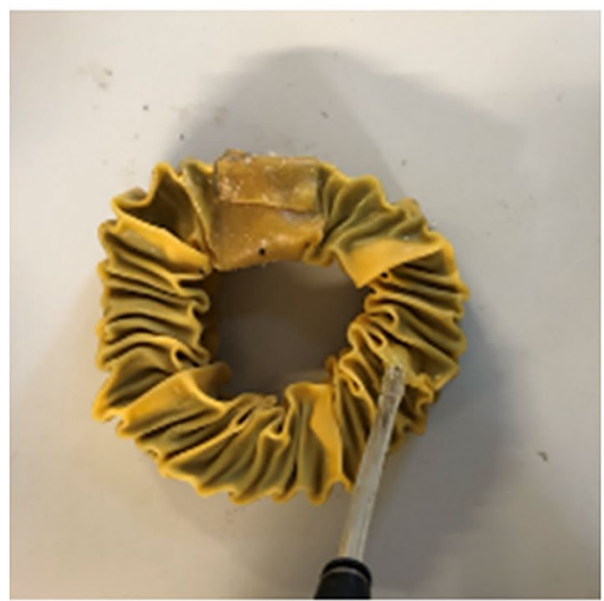

$-10 \mathrm{kPa}$

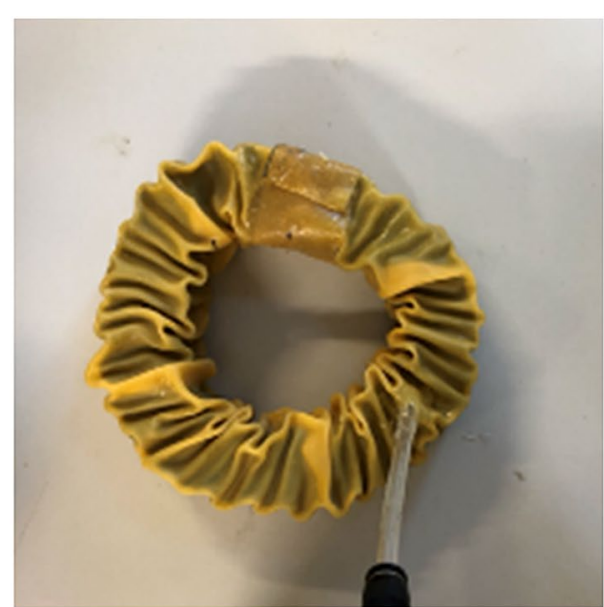

$-5 \mathrm{kPa}$

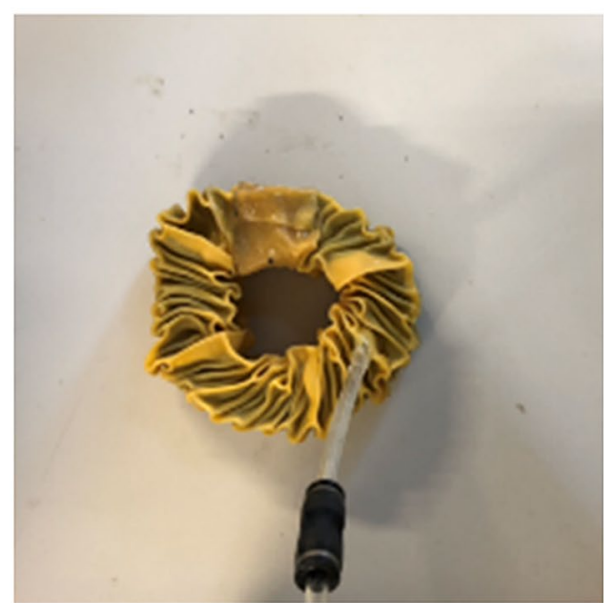

$-30 \mathrm{kPa}$

Fig. 7 Deformations of a rubber band gripper at various vacuum pressures $(0,-5,-10$, and $-30 \mathrm{kPa})$. The gripper significantly contracts even at the low vacuum pressure $(-5 \mathrm{kPa})$. The gripper significantly shrinks at a low vacuum pressure $(-5 \mathrm{kPa})$. The inner diameter of the gripper was nearly a minimum at $-30 \mathrm{kPa}$ 


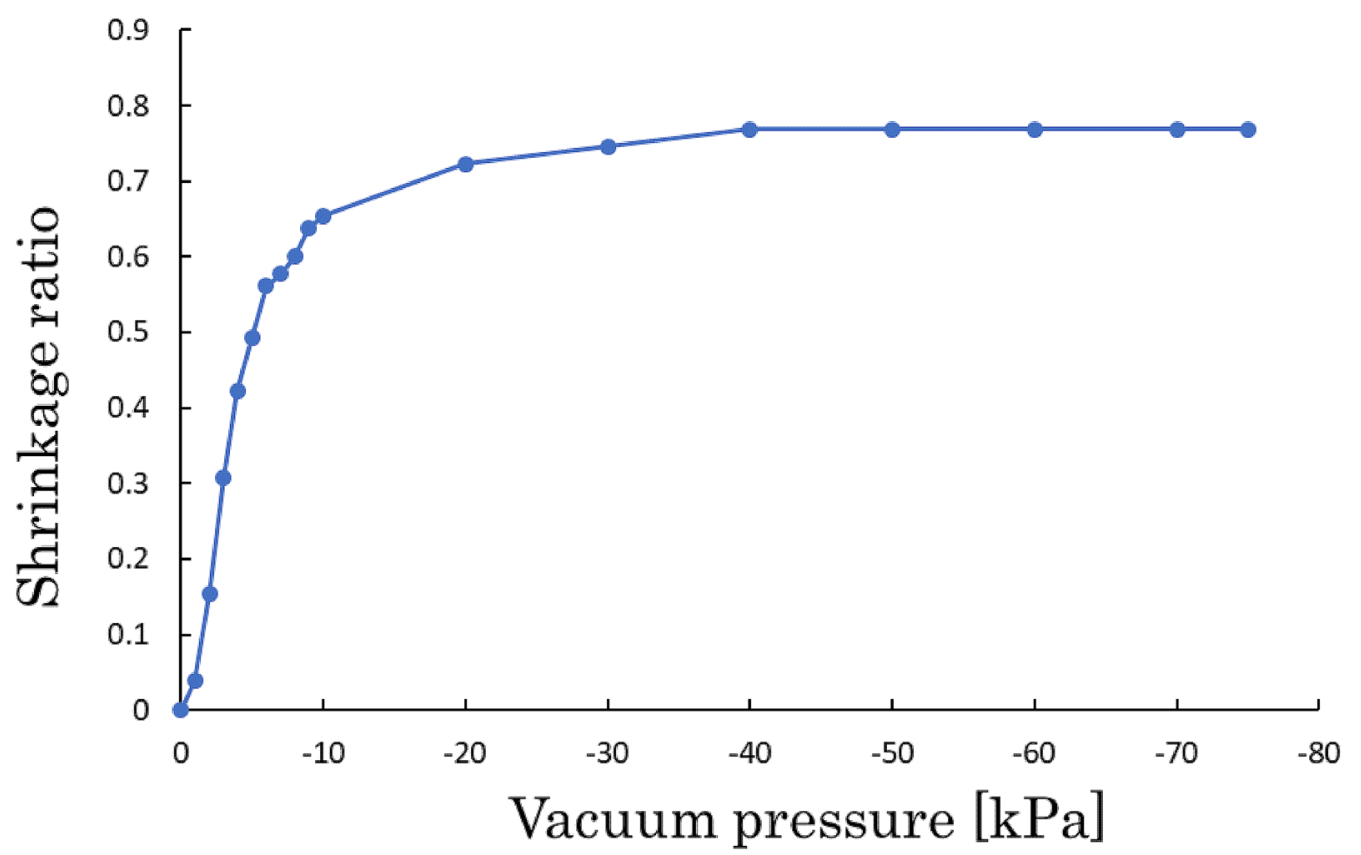

Fig. 8 Relationship between the internal air pressure and shrinkage ratio of the inner diameter of a rubber-band gripper. The gripper shrank rapidly when the inside air pressure decreased. The shrinkage ratio changed gently when the inside vacuum pressure exceeded $-10 \mathrm{kPa}$, and it became constant when the air pressure exceeded $-30 \mathrm{kPa}$

\section{Performance of encircling an object}

The wrapping performance of the gripper was assessed using a bottle of diameter $100 \mathrm{~mm}$, regular triangular prisms of sides $76 \mathrm{~mm}$ and $90 \mathrm{~mm}$, a plate of dimensions $115 \mathrm{~mm} \times 18 \mathrm{~mm}$, a cone with a base diameter of $100 \mathrm{~mm}$ and side of $70 \mathrm{~mm}$, and a cruciform prism made of three prisms. Figure 9 shows the gripper states when the internal air pressure was decreased to $-75 \mathrm{kPa}$. The gripper was able to encircle the bottle and plate without any gaps. The gripper could also encircle the triangular prisms, but small gaps were observed; larger gaps were seen for the smaller triangular prism. The gripper could wrap around the plate without any gaps; therefore, the presence of gaps is not determined by the curvature but by the angles of the corners and the peripheral length of the object. When the peripheral length of an object is small, it is difficult to encircle it when it has sharp corners because the amount of deformation of the gripper is limited when it is shrunk before contacting the object. If the peripheral length of the object is large, the gripper can encircle it even when it has sharp corners, as shown in Fig. 9.

For the cruciform prism, gaps were observed in the concave areas. The developed gripper wraps around the objects by reducing its circumference. Therefore, the gripper cannot follow the concavity of the object because the circumference needs to be extended to deform along the surface of the object. For the case of the cone, the gripper enclircled along the slope surface by twisting. To check the ability of the developed gripper to wrap around the slope, the gripper was contracted with its bottom $5 \mathrm{~mm}$ in contact with the top part of a $100 \mathrm{~mm}$ diameter pillar. Figure 10 shows this wrapping motion. The upper part of the gripper continued to contract even after the bottom of the gripper touched the pillar so that the gripper twisted by $90^{\circ}$. The inner diameter of the gripper, when twisted by $90^{\circ}$, was equal to the minimum inner diameter after contraction without encircling any objects, as shown in Fig. 7. Therefore, when the gripper is wrapped around a cone, the minimum slope angle $\theta$ that the gripper can twist by to contact the slope is determined by the minimum inner diameter of the gripper $d_{\min }$ under vacuuming, diameter of the cone with the bottom of the gripper touching $d_{c}$, and height of the gripper $h$ as follows:

$$
\theta=\cos ^{-1}\left(\frac{\boldsymbol{d}_{\boldsymbol{c}}-\boldsymbol{d}_{\text {min }}}{2 \boldsymbol{h}}\right)
$$

\section{Contact pressure}

The relationship between the contact and vacuum pressure of the gripper was examined using a pillar of 

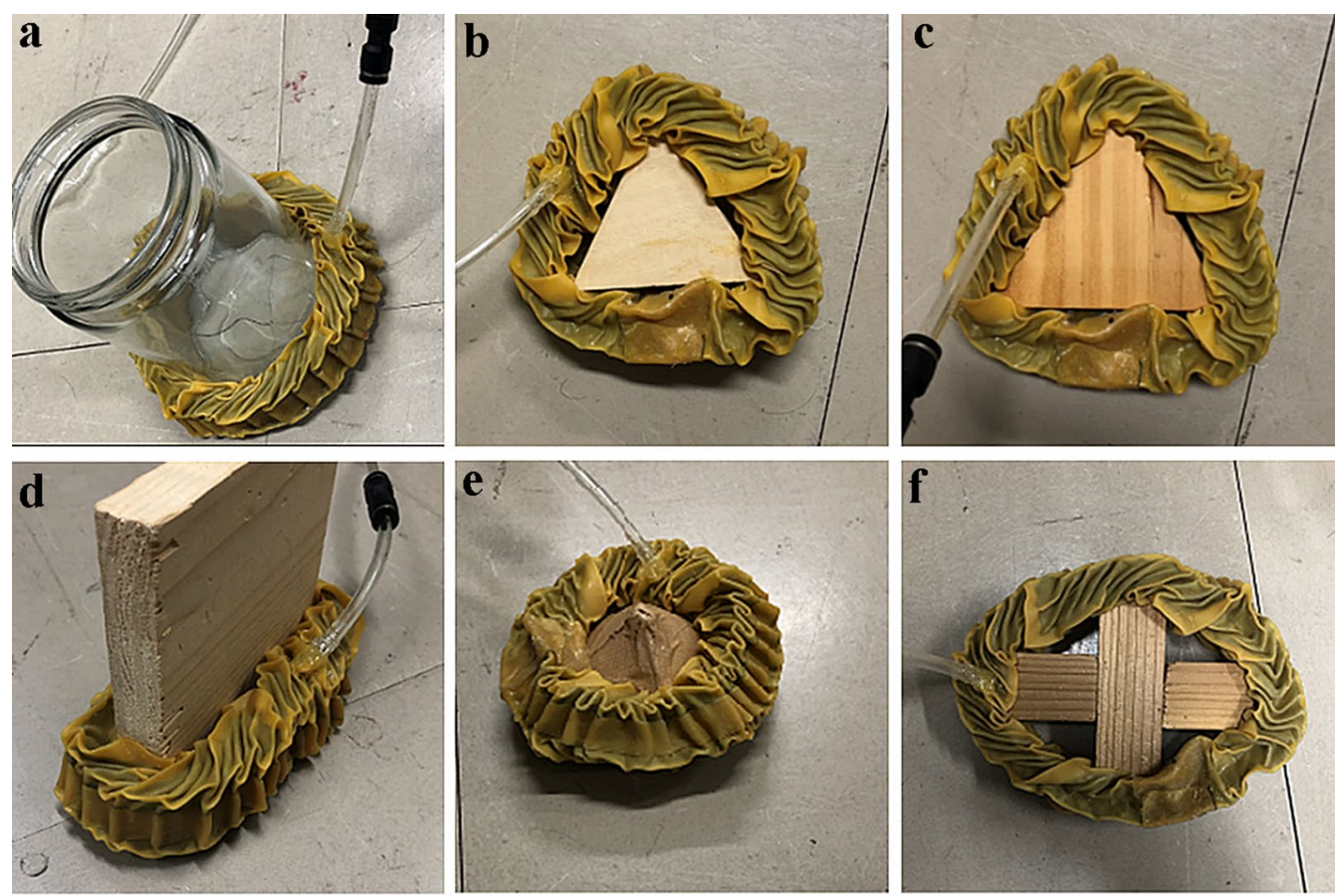

Fig. 9 Performance of gripping objects of various shapes: $\mathbf{a}$ a bottle; $\mathbf{b}$ a small triangular prism; c a large triangular prism; $\mathbf{d}$ a plate; $\mathbf{e}$ a cone; $\mathbf{f}$ a cross-shaped prism. The gripper wrapped it around the bottle, plate, and cone without any gaps. The gripper wrapped it around triangular prisms. However, larger gaps were observed for the smaller triangular prism. For a cone, the gripper twists and wraps around the slope. For a cross-shaped prism, gaps appeared in concave areas

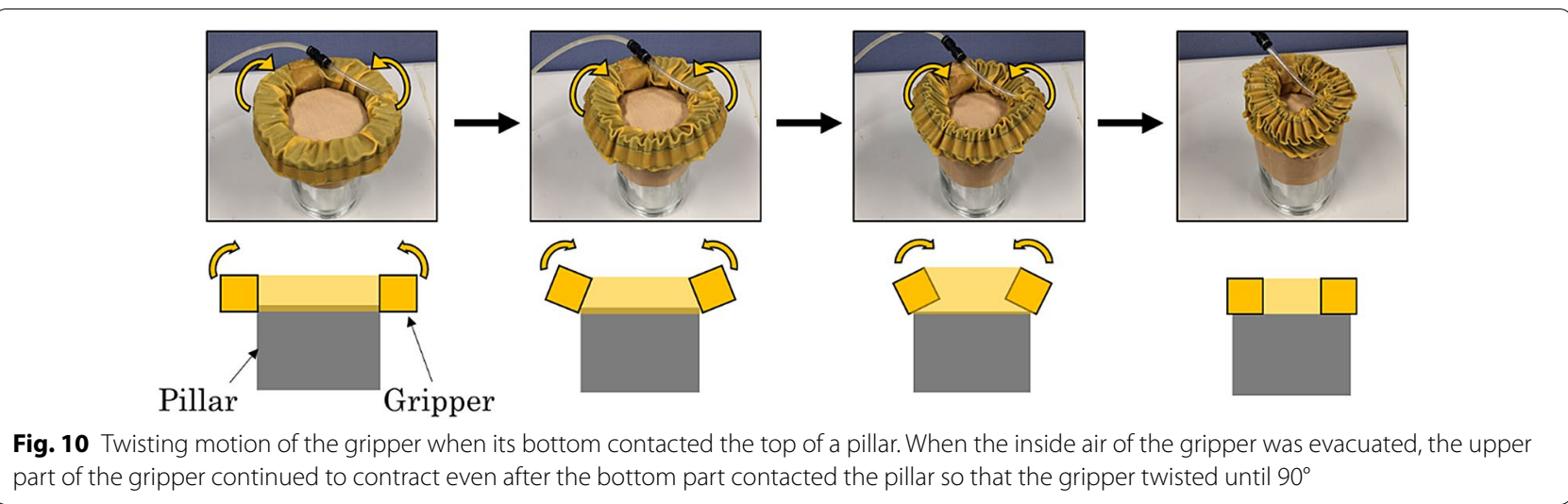

$100 \mathrm{~mm}$ diameter. The contact pressure was measured by an air-pack type contact pressure sensor (AMI3037SB-SET, AMI TECHNO, Inc.) when the internal air pressure changed from -20 to $-80 \mathrm{kPa}$ in increments of $10 \mathrm{kPa}$. The contact pressure was measured three times and averaged. As shown in Fig. 11, the contact pressure increased with the internal vacuum pressure. Because the wrapping motion of the gripper almost ends at a vacuum pressure lower than $-30 \mathrm{kPa}$, as shown in the previous section, the contact pressure can be increased by increasing the vacuum pressure. Contact pressure can also be reduced by stopping air suction when the gripper is in contact with the object. The wrapping force (i.e., the integral of contact pressure on all contact surfaces) is proportional to the contact pressure if the contact area is constant and the contact pressure is uniform. However, this assumption may not hold true if the cross-sectional shape of the object is not a circle. The future scope of the study involves an 


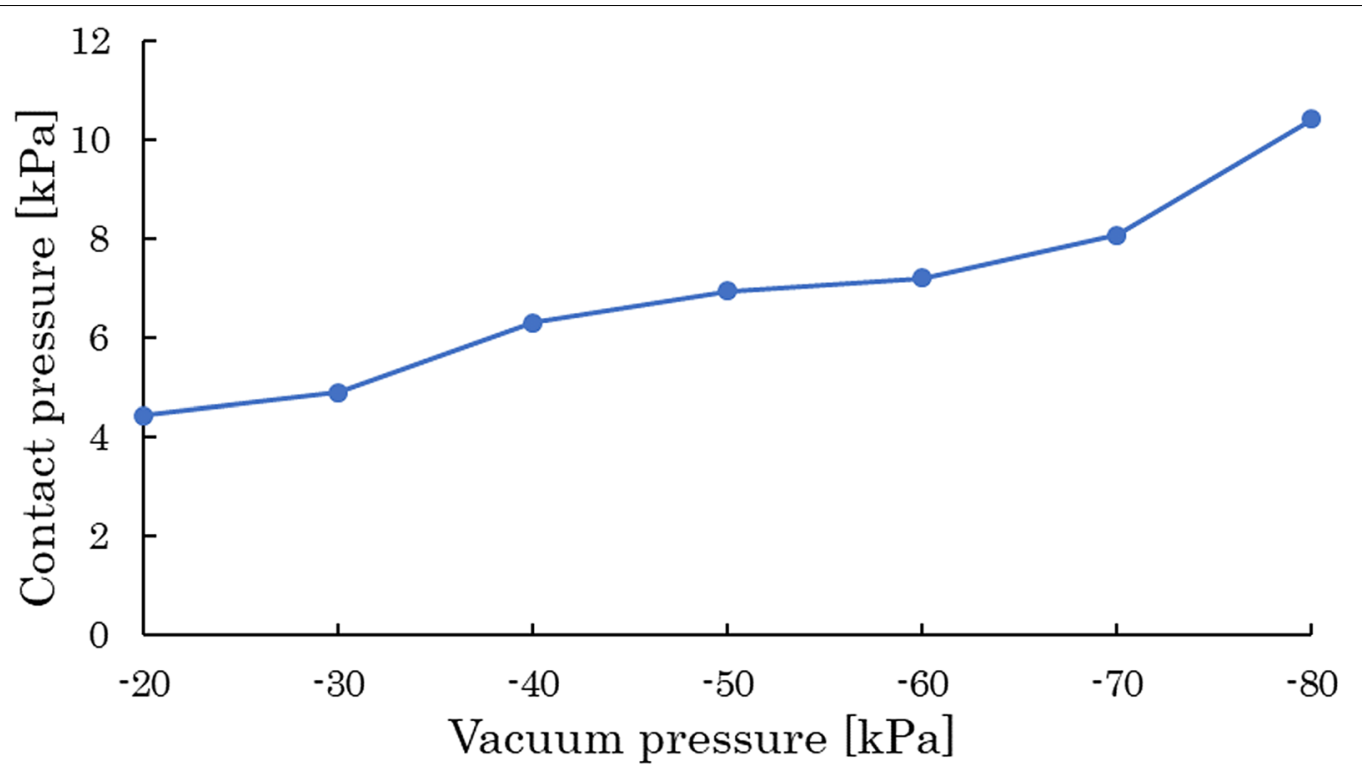

Fig. 11 Relationship between the contact pressure and internal air pressure of the rubber band gripper when wrapped around a pillar. The contact pressure increased with the internal vacuum pressure

investigation of the relationship between object shape and contact pressure.

\section{Installation on the robot arm}

The structure of the apparatus for attaching the developed gripper to a robot arm is shown in Fig. 12. The gripper and three links (195 $\mathrm{mm}$ in length) were fixed with strings (cotton, $1 \mathrm{~mm}$ diameter). The links are also fixed with strings to a metal ring of diameter $110 \mathrm{~mm}$ that is attached to the robot arm. The gripper, links, and metal ring are loosely connected to function like a ball joint. In addition, the strings are flexible so that the

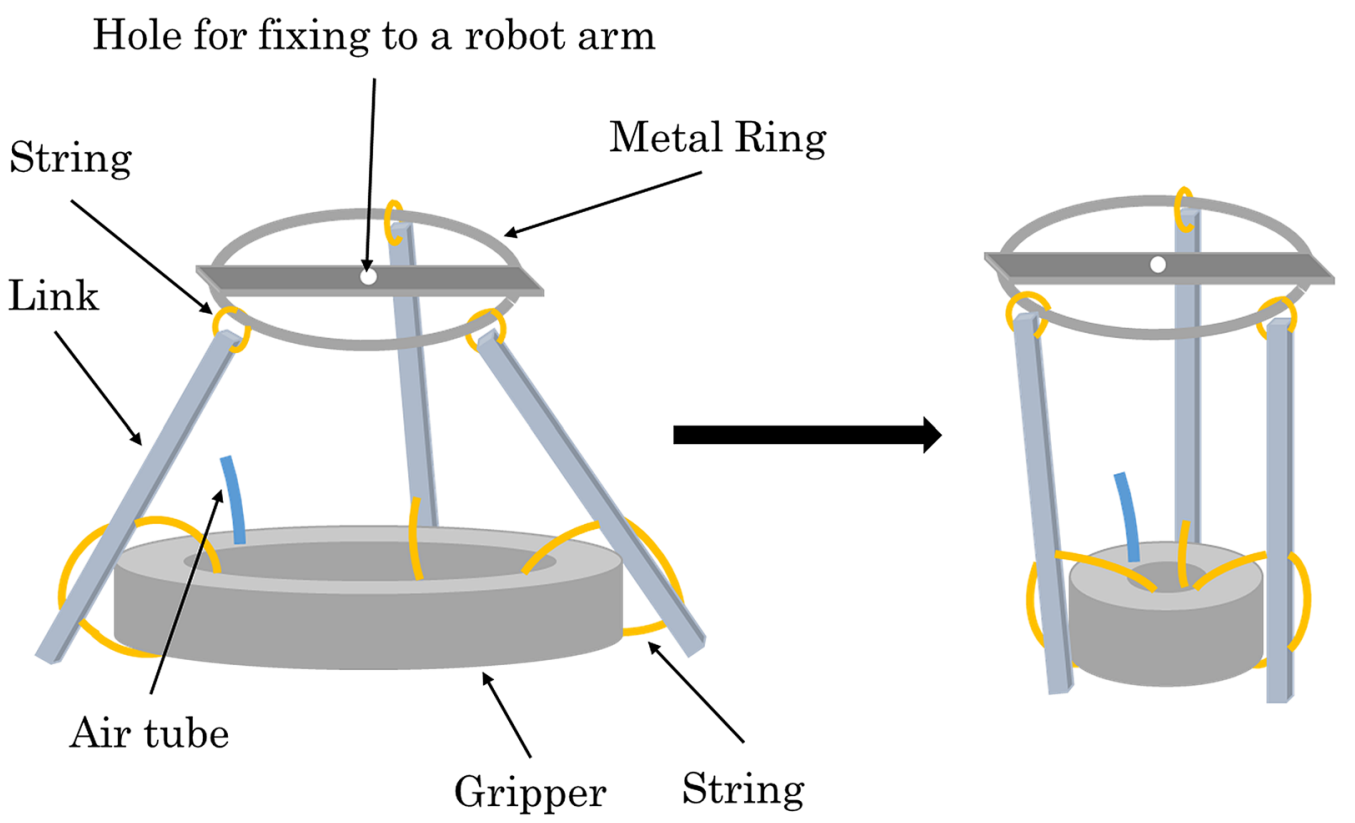

Fig. 12 Schematic illustration of the mounting device. The three links are connected with strings to the gripper and a metal ring for fixing to a robot arm. The strings are flexible so that the links move freely without interfering with the deformation of the gripper 
links move freely without interfering with the deformation of the gripper.

Figure 13 shows the method by which this apparatus is used to lift a plastic bottle (950 g), mug (289 g), toy duck (61 g), book (185 g), box (144 g), and square bread loaf $(146 \mathrm{~g})$. For the mug, the gripper grasped the handle part. The results show that the gripper fixed to a robot arm can handle objects of various shapes. For the bread, the evacuation of air was stopped at $-3 \mathrm{kPa}$ to ensure that the soft bread was not crushed when encircled without gaps. This result shows that the gripper can grasp a soft object whose cross section is not circular even though it requires control of the internal air pressure according to the contact condition.

Finally, the maximum weight that the gripper can hold was examined when grasping polyvinyl chloride pillars of $38 \mathrm{~mm}$ and $60 \mathrm{~mm}$ diameter, a glass bottle of $100 \mathrm{~mm}$ diameter, and a steel quadrangular prism
$(12 \mathrm{~mm} \times 100 \mathrm{~mm})$. The maximum weight that the gripper could handle without slippage was $2.4,3.7,3.3$, and $3.0 \mathrm{~kg}$, respectively. The maximum weight depends on the shape and material of the items; nonetheless, the results achieved indicate that the developed gripper is capable of holding heavy objects.

\section{Conclusions}

In this study, a flexible ring-shaped gripper that wraps around an object like a rubber band was developed by evacuating the air inside the gripper. Experimental results showed that the gripper could wrap around objects of various shapes, such as bottles, boxes, cones, and plates without any gaps, owing to the flexibility of the sponges and the outer membrane. Encircling an object without any gaps increases the contact area and decreases the contact pressure. Therefore, the developed

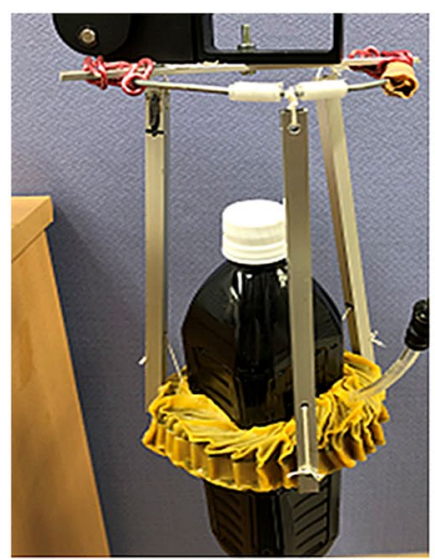

$\mathbf{a}$

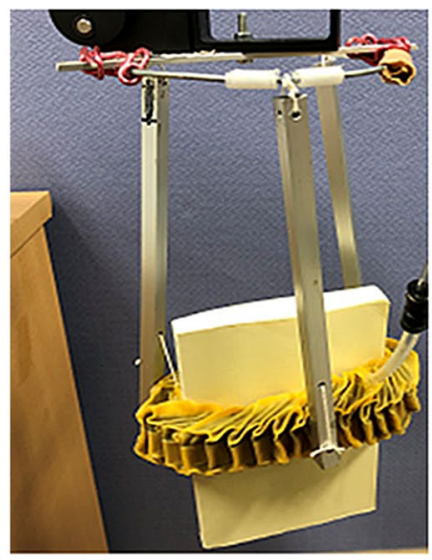

d

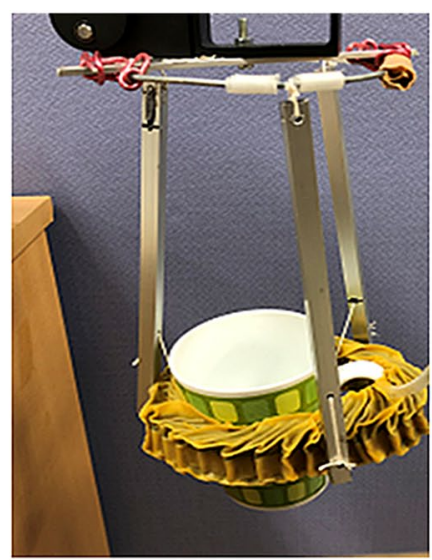

b

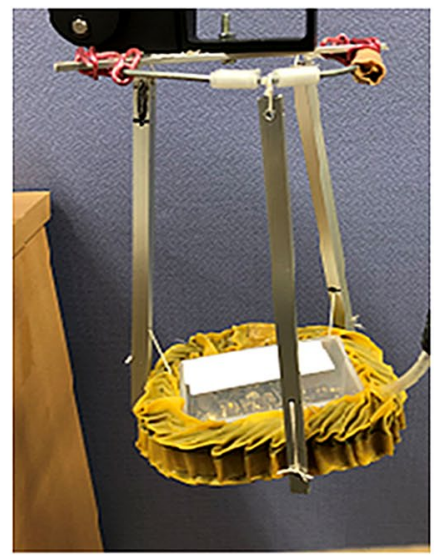

e

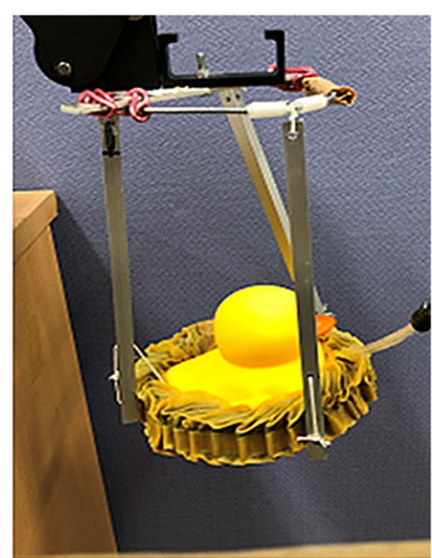

c

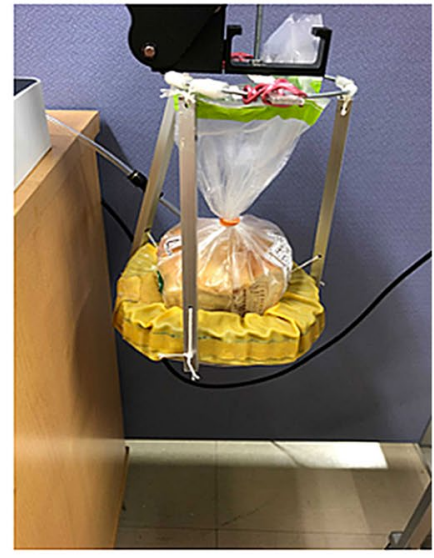

f

Fig. 13 The gripper picks up various objects a a bottle (950 g); b a mug (289 g); c a duck toy (61 g); d a book (185 g); e a box (144 g); f square bread loaf $(146 \mathrm{~g})$. The gripper was able to lift objects of various shapes by wrapping around them. For the bread, exhausting the inside air of the gripper was stopped at $-3 \mathrm{kPa}$ to avoid crashing after the gripper wrapped the square bread without gaps 
gripper can stably grasp objects without considering the point of contact, which is required for a finger-shaped one. In this work, we have also demonstrated that a robot arm with a gripper can lift a heavy object. Increasing the internal air pressure of the gripper increased its wrapping force; at the same time, the gripper was able to grasp soft bread without crushing it by decreasing the internal air pressure.

The above observations suggest the following possibilities for future work. First, the developed gripper cannot achieve contact with the concavity of an object because the gripper shrinks by shortening its circumferential length. To fit the concavity, the length of the gripper needs to be extended partially. A larger contact area with the object is appropriate for grasping a fragile object. Therefore, developing a structure that can deform for indentation is a challenging direction of study.

Next, the developed mounting apparatus had flexible strings connecting the links to the gripper. This method allowed flexible deformation of the gripper but caused swaying of the gripped object because the links were always free to move. The rocking motion should be minimized for positioning the object precisely. In addition, the connecting method limits the use of the gripper to hang objects because gravity causes the gripper to position only objects that hang downwards. If the gripper can follow the posture of the robot arm, it could be used for a wider range of applications.

Finally, a theoretical analysis of the gripper's deformation is an important consideration. It is necessary to investigate how the sponge and outer membrane interact with each other during contraction of the gripper. This study shows that the developed gripper can wrap around an object with a slope. The maximum inclinations of such slopes were analyzed for a cone but not for other objects, such as triangular pyramids. Analysis of wrapping objects in three dimensions is therefore required.

\section{Authors' contributions}

The first author conducted the study under the supervision of the second author. The first and second authors wrote the manuscript. Both authors read and approved the final manuscript.

\section{Funding}

This work was supported by JSPS KAKENHI Grant Number JP18K04064.

\section{Availability of data and materials}

Not applicable.

\section{Declarations}

\section{Competing interests}

The authors declare no competing financial interests.

\section{Author details}

${ }^{1}$ Graduate School of Information Science and Engineering, Ritsumeikan University, 1-1-1 Noji-Higashi, Kusatsu-shi, Shiga 525-8577, Japan. ${ }^{2}$ College of Information Science and Engineering, Ritsumeikan University, Kusatsu-shi, Japan.

Received: 25 December 2020 Accepted: 7 April 2021

Published online: 26 April 2021

\section{References}

1. Laschi C, Mazzolai B, Cianchetti M (2016) Soft robotics: technologies and systems pushing the boundaries of robot abilities. Sci Robot. https://doi. org/10.1126/scirobotics.aah3690

2. Shintake J, Cacucciolo V, Floreano D, Shea H (2018) Soft Robotic gripper. Adv Mater 30:1707035. https://doi.org/10.1002/adma.201707035

3. Suzumori K, likura S, Tanaka H (1991) Development of flexible microactuator and its apolypropylenelications to robotic mechanisms. In: Proceedings 1991 IEEE International Conference on Robotics and Automation, Sacramento, CA, USA. https://doi.org/10.1109/ROBOT.1991.131850

4. Zhou J, Chen S, Wang Z (2017) A soft-robotic gripper with enhanced object adaptation and grasping reliability. IEEE Robot Autom Lett 2(4):2287-2293. https://doi.org/10.1109/LRA.2017.2716445

5. Wang Z, Torigoe Y, Hirai S (2017) A prestressed soft gripper: Design, modeling, fabrication, and tests for food handling. IEEE Robot Autom Lett 2(4):1909-1916

6. Wang Z, Or K, Hirai S (2020) A dual-mode soft gripper for food packaging. Robot Autonom Syst 125:103427. https://doi.org/10.1016/j.robot.2020. 103427

7. NITTA SOFTmatics https://www.nitta.co.jpolypropyleneroduct/robot hand/ Accessed: Nov 2020

8. Soft Robotics Inc Soft Robotics, https://www.softroboticsinc.com/ Accessed: Nov 2020

9. Wei Y, Chen Y, Ren T et al (2016) A novel, variable stiffness robotic gripper based on integrated soft actuating and particle jamming. Soft Robot 3(3):134-143. https://doi.org/10.1089/soro.2016.0027

10. Hao Y, Wang T, Xie Z et al (2018) A eutectic-alloy-infused soft actuator with sensing, tunable degrees of freedom, and stiffness properties. J Micromech Microeng 28(2):024004. https://doi.org/10.1088/1361-6439/ aa9d0e

11. Jiang $P$, Yang $Y$, Chen $M$ et al (2019) A variable stiffness gripper based on differential drive particle jamming. Bioinspir Biomimetics 14(3):036009. https://doi.org/10.1088/1748-3190/ab04d1

12. Fei Y, Wang J, Pang W (2019) A novel fabric-based versatile and stiffnesstunable soft gripper integrating soft pneumatic fingers and wrist. Soft Robot 6(1):1-20. https://doi.org/10.1089/soro.2018.0015

13. Mitsuda T, Otsuka S (2021) Active bending mechanism employing granular jamming and vacuum-controlled adaptable gripper. IEEE Robot Autom Lett 6(2):3041-3048. https://doi.org/10.1109/LRA.2021.3058914

14. Brown E, Rodenberg N, Amend J et al (2010) Universal robotic gripper based on the jamming of granular material. PNAS 44:18809-18814. https://doi.org/10.1073/pnas.1003250107

15. Mitsuda T, Kuge S, Wakabayashi M et al (2002) Wearable force display using a particle mechanical constraint. Presence 11(6):569-577. https:// doi.org/10.1162/105474602321050703

16. Li S, Stampfli JJ, Xu HJ et al. (2019) A vacuum-driven origami "magic-ball" soft gripper. In: IEEE International Conference on Robotics and Automation, 2206-2213

17. Bridgestone Air Picker and Air Gripper https://www.bridgestoneengi neered.com/Air_Picker_and_Air_Gripper/ Accessed: March 2021

18. Wang Z, Kanegae R, Hirai S (2020) Circular shell gripper for handling food products, soft robotics. Soft Robot. https://doi.org/10.1089/soro.2019. 0140

\section{Publisher's Note}

Springer Nature remains neutral with regard to jurisdictional claims in published maps and institutional affiliations. 